\title{
Remarks on Some Existence Theorems for Optimal Control ${ }^{2}$
}

\author{
L. Cesari, ${ }^{2}$ J. R. LA PALm, ${ }^{3}$ AND T. NishiURA ${ }^{4}$
}

\begin{abstract}
First, a remark is made that a growth condition contained in previous papers by Cesari concerning existence theorems for optimal controls can be replaced by a slightly more general condition. In this more general condition, a constant $M_{\epsilon} \geqslant 0$ is replaced by any function $M_{\epsilon}(t) \geqslant 0$ which is assumed to be $L$-integrable in every finite interval.

Then, the remark is made that the same condition, which is usually required to be satisfied by the functions $f_{0}(t, x, u), f(t, x, u)$ characterizing the control, can be required to be satisfied only by the admissible pairs $x(t), u(t)$ of the class $\Omega$ in which the optimum is being sought. This generalization requires a subtle argument. The new condition parallels now the usual conditions of the type $\int\left|x^{\prime}\right|^{p} d t \leqslant M$, which are required to be satisfied by the admissible pairs of the class $\Omega$.
\end{abstract}

\section{Introduction}

In a previous paper (Ref. 1), the use of growth conditions in the proof of existence theorems for optimal control with unbounded control spaces was analyzed, and weaker alternate hypotheses were proposed for the various parts of the argument. In the present paper, a few more remarks on growth conditions are added to those in Ref. 1.

\section{Growth Conditions}

In Ref. 2, it was shown that Ascoli's selection theorem can be replaced by Helly's selection theorem on certain components, and this remark was later

\footnotetext{
${ }^{3}$ Paper received October 29,1968 . The work of $L$. Cesari was partially supported by the Office of Scientific Research, United States Air Force, Grant No. 942-65. The work of T. Nishiura was partially supported by the National Science Foundation, Grant No. GP-7676.

${ }^{2}$ Professor of Mathematics, University of Michigan, Ann Arbor, Michigan.

${ }^{3}$ Assistant Professor of Mathematics, Wayne State University, Detroit, Michigan.

${ }^{4}$ Professor of Mathematics, Wayne State University, Detroit, Michigan.
} 
used systematically in Refs. $1,3,4$. If $x^{1}, \ldots, x^{\alpha}, 0 \leqslant \alpha \leqslant n$, are the components of the trajectories $x=\left(x^{1}, \ldots, x^{n}\right)$ on which Ascoli's selection theorem is applied, then one must show that the same components for the trajectories of a minimizing sequence are equiabsolutely continuous. We proved in Ref. 1 that the following growth condition $\left(\gamma_{i}\right), i=1, \ldots, \alpha$, concerning the growth of $f_{i}(t, x, u)$ with respect to the nonnegative comparison function $H(t, x, u)$ can be used to that effect: $\left(\gamma_{i}\right)$ Given $\epsilon>0$, there is some nonnegative constant $M_{\epsilon}$, which may depend on $\epsilon$, such that

$$
\left|f_{i}(t, x, u)\right| \leqslant M_{i \epsilon}+\epsilon H(t, x, u), \quad(t, x, u) \in M .
$$

Here, we use the same notation as in Ref. 1 , where $M$ is a closed subset of the $t x u$-space $E_{1+n+m}$ whose projection on the $t$-axis is compact. Let $[a, b]$ be an interval of the $t$-axis such that $M$ is completely contained in the slab $a \leqslant t \leqslant b, x \in E_{n+m}$. Condition $\left(\gamma_{i}\right)$ can be replaced by the following slightly more general hypothesis: $\left(\gamma_{i}^{\prime}\right)$ Given $\epsilon>0$, there is some nonnegative function $M_{\epsilon}(t)$, which may depend on $\epsilon$, which is $L$-integrable in $[a, b]$ and such that

$$
\left|f_{i}(t, x, u)\right| \leqslant M_{i \epsilon}(t)+\epsilon H(t, x, u) \text { for almost all } t \in[a, b] \text { and }(t, x, u) \in M .
$$

Note that it is enough to know that Condition $\left(\gamma_{i}\right)$, or Condition $\left(\gamma_{i}^{\prime}\right)$, is verified for all $\epsilon$ of a given countable family [ $\epsilon$ ] of positive numbers $\epsilon$, which is dense at $\epsilon=0$.

\section{Statement of Existence Theorem}

In Ref. 1 , it was mentioned that Condition $\left(\gamma_{i}\right)$ could be replaced by a usual $L_{p}$-type condition on the trajectories $x(t)$ of the class $\Omega$ of trajectories on which the minimum is sought. Such condition simply requires that: $\left(\delta_{i}\right)$ There are constants $p_{i}>1$ and $M_{i} \geqslant 0$ such that, for every trajectory $x(t)$, $i_{1} \leqslant t \leqslant t_{2}$, of the class $\Omega$, we have

$$
\left.\int_{t_{1}}^{t_{3}}\left|d x^{i}\right| d t\right|^{p_{i}} d t \leqslant M_{i}
$$

Condition $\left(\gamma_{i}\right)$ can be reworded as a condition on $\Omega$ with a slight gain in generality, and then Conditions $\left(\gamma_{i}\right)$ and $\left(\delta_{i}\right)$ are expressed in similar forms [see Condition $\left(\epsilon_{i}\right)$ below]. Finally, there is also a slight gain in generality by considering more than one comparison function $H$. For the sake of clarity, we reword Existence Theorem A of Ref. 1 under the new hypotheses. We use the same definition of admissible pair $x(t), u(t), t_{1} \leqslant t \leqslant t_{2}$, as in Ref. $1, \S 4$, No. 1 . 
Existence Theorem. Let $\alpha, \beta, \gamma, n, 0 \leqslant \beta \leqslant \alpha \leqslant n, 1 \leqslant \gamma$, be given integers, and for $x=\left(x^{1}, \ldots, x^{n}\right)$, let $y, z$ denote $y=\left(x^{1}, \ldots, x^{\alpha}\right), z=\left(x^{\alpha+1}, \ldots, x^{n}\right)$, so that $x=(y, z)$. Let $A_{0}$ be a compact subset of the $t y$-space $E_{\alpha+1}$, let $[a, b]$ be a closed interval of the $t$-axis such that $a \leqslant t \leqslant b$ for all $(t, y) \in A_{0}$, and let $I=\left[a_{\alpha+1}, b_{\alpha+1}\right] \times \cdots \times\left[a_{n}, b_{n}\right]$ be a finite closed interval of the $z$-space $E_{n-\alpha}$; thus, $A=A_{0} \times I$ is a compact subset of the $t x$-space $E_{n+1}$. For every $(t, y) \in A_{0}$, let $U(t, y)$ be a given closed subset of the $u$-space $E_{m}$ satisfying property $(U)$ in $A_{0}$. Let $M_{0}$ be the set of all $(t, y, u)$ with $(t, y) \in A_{0}$, $u \in U(t, y)$; then, $M=M_{0} \times I$ is the set of all $(t, x, u)$ with $(t, x) \in A$, $u \in U(t, y), x=(y, z)$. Let $f(t, y, u)=\left(f_{1}, \ldots, f_{n}\right), H(t, y, u)=\left(H_{1}, \ldots, H_{\gamma}\right)$ be functions defined on $M_{0}$, and assume that $f_{1}, \ldots, f_{\alpha}$ are continuous on $M_{0}$ and that $H_{1}, \ldots, H_{y}, f_{\alpha+1}, \ldots, f_{n}$ are nonnegative and lower semicontinuous on $M_{0}$. For every $(t, y) \in A_{0}$, let $Q_{H}(t, y)$ be the set of all

$$
\tilde{z}=\left(3^{1}, \ldots, 3^{\eta}, z^{1}, \ldots, z^{n}\right) \in E_{n+\gamma}
$$

defined by

$$
\begin{gathered}
Q_{H}(t, y)=\left[\tilde{z} \mid 3^{s} \geqslant H_{s}(t, y, u), \quad s=1, \ldots, \gamma, \quad z^{i}=f_{i}(t, y, u), \quad i=1, \ldots, \alpha,\right. \\
\left.z^{i} \geqslant f_{i}(t, y, u), \quad i=\alpha+1, \ldots, n, \quad u \in U(t, y)\right] \subset E_{n+\gamma}
\end{gathered}
$$

and assume that $Q_{H}(t, y)$ is convex for every $(t, y) \in A_{0}$ and satisfies property $(Q)$ in $A_{0}$. For every $(t, x, u) \in M, x=(y, z)$, we write $f(t, x, u)=f(t, y, u)$, $H(t, x, u)=H(t, y, u), U(t, x)=U(t, y), Q_{H}(t, x)=Q_{H}(t, y)$. Let $B$ be a closed subset of the $t_{1} x_{1} t_{2} x_{2}$-space $E_{2 n+2}, x_{1}=\left(x_{1}{ }^{1}, \ldots, x_{1}{ }^{n}\right), x_{2}=\left(x_{2}{ }^{1}, \ldots, x_{2}{ }^{n}\right)$, and assume that $B$ is independent of $x_{2}^{\alpha+1}, \ldots, x_{2}{ }^{n}$; hence, $B$ is of the form $B=B_{0} \times E_{n-\alpha}$, where $B_{0}$ is a closed subset of $E_{n+2+\alpha}$. Let $e\left(t_{1}, x_{1}, t_{2}, x_{2}\right)$ be a real-valued continuous function defined on $B$, which is monotone nondecreasing with respect to each variable $x_{2}^{\alpha+1}, \ldots, x_{2}{ }^{n}$. Let $M_{s} \geqslant 0$, $s=1, \ldots, \gamma$, and $M_{i} \geqslant 0, p_{i}>1, i=1, \ldots, \beta$, be given constants. Let [ $\left.\epsilon\right]$ be a given family of positive numbers which is dense at $\epsilon=0$; and, for every $i=\beta+1, \ldots, \alpha$ and $\epsilon \in[\epsilon]$, let $M_{i \epsilon}(t), a \leqslant t \leqslant b$, be a given real-valued, nonnegative, $L$-integrable function in $[a, b]$, and let $s=s(i)$ be any one of the numbers $s=1, \ldots, \gamma$. Let $\Omega$ be the class of all admissible pairs $x, u$ (defined as usual relative to the sets $A, U(t, x), M, B$ and functions $f$ and $e$ ) for which $\left(\epsilon_{i}\right)$

$$
\begin{aligned}
& \int_{t_{1}}^{t_{2}} H_{s}(t, y(t), u(t)) d t \leqslant M_{s}, \quad s=1, \ldots, \gamma, \\
& \int_{t_{1}}^{t_{2}}\left|f_{i}(t, y(t), u(t))\right|^{p_{i}} d t \leqslant M_{i}, \quad i=1, \ldots, \beta,
\end{aligned}
$$


and

$$
\begin{gathered}
\left|f_{i}(t, y(t), u(t))\right| \leqslant M_{i \epsilon}(t)+\epsilon H_{s}(t, y(t), u(t)), \\
s=s(i), \quad i=\beta+1, \ldots, \alpha, \quad \text { and almost all } t \in\left[t_{1}, t_{2}\right] .
\end{gathered}
$$

Let us assume that $\Omega$ is nonempty. Then, the functional

$$
I[x, u]=e\left(t_{1}, x\left(t_{1}\right), t_{2}, x\left(t_{2}\right)\right)
$$

has an absolute minimum in $\Omega$.

\section{Remarks and Examples}

If $A$ is not compact, then, as usual (see Refs. 2 and 4 ) the theorem above can be applied after it is shown that a minimizing sequence is certainly contained in some compact subset $A_{0}$ of $A$.

If one or more of the functions $f_{i}, i=\alpha+1, \ldots, n_{3}$ coincide with corresponding functions $H_{s}$, then the repetitions in the definition of $Q_{H}$ can be disregarded and it suffices to consider sets $Q_{H}$ of lower dimension, as already mentioned in Ref. 1 in a particular case.

Remark 4.l. If Condition $\left(\gamma_{i}^{\prime}\right)$ is satisfied for $i=\beta+1, \ldots, \alpha$, that is, in the present context, if

$$
\mid f_{i}(t, y, u)_{1} \leqslant M_{i \mathrm{\epsilon}}(t)+\epsilon H_{s}(t, y, u), \quad i=\beta+1, \ldots, \alpha, \quad s=s(i), \quad \epsilon \in[\epsilon],
$$

for all $(t, y, u) \in M_{0}$, then certainly the relations (4) are satisfied by all admissible pairs. The interest of the present existence theorem lies in the fact that the relations (5) may not hold for all $(t, y, u) \in M_{0}$, and the relations (4) represent an actual restriction in the class of admissible pairs which we allow to belong to $\Omega$. This can be seen by the following example. Take $n=2$, $m=1, \beta=0, \alpha=1, \gamma=1, H=H_{1}(t, y, u)=u^{2}, f_{1}=f_{1}(t, y, u)=u$, $f_{2}=f_{2}(t, y, u)=u^{2}, u \in U=(-\infty, \infty)$. The set $Q_{H}$ is now the set of all $z=\left(z^{1}, z^{2}, z^{3}\right)$ with $z^{1}=u, z^{2} \geqslant u^{2}, z^{3} \geqslant u^{2}$. This set is certainly convex and has property $(Q)$. Now, let $[\epsilon]=\{\epsilon \mid 0<\epsilon \leqslant 1\}$; and, for $i=1, \epsilon \in[\epsilon]$, let $M_{i \epsilon}(t)=10^{-2} \epsilon^{-2}, a \leqslant t \leqslant b$, a constant. Then, the relation $\left|f_{1}\right| \leqslant$ $M_{1 \epsilon}(t)+\epsilon H_{1}$ becomes

$$
|u| \leqslant 10^{-2} \epsilon^{-2}+\epsilon u^{2}
$$


and this relation must be satisfied for all $\epsilon, 0<\epsilon \leqslant 1$. Obviously, $u= \pm 10^{-1}$ does not satisfy $(\gamma)$ for $\epsilon=1$. Denote by $U^{\prime}(t, y)$ the set of those $u \in U(t, y)$ for which the relation $(\gamma)$ holds for all $\epsilon, 0<\epsilon \leqslant 1$. We can prove that $U^{\prime}(t, y)$ is the union of three disjoint intervals $\left(-\infty,-\beta_{0}\right],\left[-\alpha_{0}, \alpha_{0}\right],\left[\beta_{0}, \infty\right)$, where $0<\alpha_{0}<\beta_{0}$. A consequence of this fact is that the subset of $Q_{H}(t, y)$ determined by $U^{\prime}(t, y)$ is not convex, that is, the set

$$
\left\{\tilde{z}=\left(z^{1}, z^{2}, z^{3}\right) \mid z^{1}=u, \quad z^{2} \geqslant u^{2}, \quad z^{3} \geqslant u^{2}, \quad u \in U^{\prime}(t, y)\right\}
$$

is not convex.

To prove our statement about $U^{\prime}(t, y)$, consider $u \geqslant 0$. Then, $(\gamma)$ is equivalent to the relation $\epsilon u^{2}-u+10^{-2} \epsilon^{-2} \geqslant 0$. Checking the discriminant of the quadratic, we find that only $\epsilon \in\left[25^{-1}, 1\right]$ need be considered. The quadratic formula yields

$$
u \geqslant\left\{1+\left[1-(25 \epsilon)^{-1}\right]^{1 / 2}\right\} / 2 \epsilon
$$

or

$$
0 \leqslant u \leqslant\left\{1-\left[1-(25 \epsilon)^{-1}\right]^{1 / 2}\right\} / 2 \epsilon
$$

for $25^{-1} \leqslant \epsilon \leqslant 1$. Due to the continuity of $\left\{1 \pm\left[1-(25 \epsilon)^{-1}\right]^{1 / 2}\right\} / 2 \epsilon$ over the closed interval $\left[25^{-1}, 1\right]$, there are constants $\alpha_{0}$ and $\beta_{0}, 0<\alpha_{0}<\beta_{0}$, such that $u \geqslant 0$ satisfies the relation $(\gamma)$ for all $\epsilon \in\left[25^{-1}, 1\right]$ if, and only if, $u \geqslant \beta_{0}$ or $u \leqslant \alpha_{0}$. Consequently, $u \geqslant 0$ satisfies the relation $(\gamma)$ for all $\epsilon, 0<\epsilon \leqslant 1$, if, and only if, $u \geqslant \beta_{0}$ or $u \leqslant \alpha_{0}$. By a symmetric argument, we find that

$$
U^{\prime}(t, y)=\left\{u|| u \mid \leqslant \alpha_{0} \quad \text { or } \quad|u| \geqslant \beta_{0}\right\} \text {. }
$$

Remark 4.2. The sets $Q_{H}(t, y)$ of the existence theorem certainly satisfy property $(Q)$ if they are convex and if the following growth condition is satisfied: $(\alpha) 1$ is of slower growth than $H_{1}, \ldots, H_{\gamma}$, and each $f_{i}$ is of slower growth than $H_{s(i)}, i=1, \ldots, \alpha$. In other words, given $\epsilon>0$, there is some constant $\bar{u}_{\epsilon} \geqslant 0$ such that $(t, y) \in A_{0}, u \in U(t, y),|u| \geqslant \bar{u}_{\epsilon}$ implies that

$$
\begin{gathered}
1 \leqslant \epsilon H_{j}(t, y, u), \quad j=1, \ldots, \gamma, \\
\left|f_{i}(t, y, u)\right| \leqslant \epsilon H_{s(i)}(t, y, u), \quad i=1, \ldots, \alpha .
\end{gathered}
$$

The proof is analogous to the one given in Ref. 1, $\$ 2$, No. 2 .

Example 4.1. Let us consider the well-known control problem with differential equations $d x / d t=u, d y / d t=t^{\alpha} u^{2}, 0 \leqslant t \leqslant 1$, boundary conditions $x(0)=1, y(0)=0, x(1)=0$, control space $U=(-\infty<u<\infty)$, 
and functional $I=g=y(1)$. Here, $\alpha$ is a constant, $0<\alpha<1$. We seek the minimum of $I$ in the class $Q$ of all admissible systems $x(t), y(t), u(t), 0 \leqslant t \leqslant 1$. This class $\Omega$ is nonempty since $x(t)=1-t, y(t)=(\alpha+1)^{-1} t^{\alpha+1}, u(t)=-1$, $0 \leqslant t \leqslant 1$, is an admissible system. To show that we can apply the existence theorem above, let us take $H(t, y, u)=H=t^{\alpha} u^{2}, M_{\epsilon}(t)=\epsilon^{-1} t^{-\alpha}$, and let us prove that $|u| \leqslant \epsilon^{-1} t^{-\alpha}+\epsilon t^{\alpha} u^{2}$ for all $u \in U, 0<t \leqslant 1,0<\epsilon \leqslant 1$. Indeed, for $|u| \geqslant \epsilon^{-1} t^{-\alpha}$, we have $|u|=|u|^{-1} u^{2} \leqslant \epsilon t^{\alpha} u^{2} \leqslant M_{\epsilon}(t)+\epsilon H$; for $|u| \leqslant \epsilon^{-1} t^{-\alpha}$, we have $|u| \leqslant \epsilon^{-1} t^{-\alpha} \leqslant M_{\epsilon}(t)+\epsilon H$. Thus, here condition $\left(\gamma_{i}^{\prime}\right)$ holds for $i=1$. For the minimum of $I$, it suffices to consider the subclass $\Omega_{0}$ of all systems $x, y, u$ of $\Omega$ with $0 \leqslant I=y(1) \leqslant(\alpha+1)^{-1}$; hence,

$$
\int_{0}^{1} H d t=\int_{0}^{1} t^{\alpha} u^{2} d t=y(1) \leqslant(\alpha+1)^{-1}=\eta .
$$

Then, $0 \leqslant y(t) \leqslant y(1) \leqslant \eta$, and, if $\epsilon=1$, also

$$
\begin{aligned}
|x(t)| & =\left|1+\int_{0}^{t} u(\tau) d \tau\right| \leqslant 1+\int_{0}^{1}\left(\tau^{-\alpha}+\tau^{\alpha} u^{2}\right) d \tau \\
& \leqslant 1+(1-\alpha)^{-1}+(\alpha+1)^{-1}=\delta .
\end{aligned}
$$

Thus, we can restrict ourselves to the compact subset $A_{0}$ of the txy-space

$$
A_{0}=[0 \leqslant t \leqslant 1,|x| \leqslant \delta, 0 \leqslant y \leqslant \eta] .
$$

The conditions of the existence theorem are all satisfied with $n=2, \alpha=1$, $\beta=0, \gamma=1$, and the problem above possesses an absolute minimum.

Example 4.2. As a second example, let us consider the control problem with differential equations $d x / d t=u, d y / d t=t u^{2}, 0 \leqslant t \leqslant 1$, boundary conditions $x(0)=1, y(0)=0, x(1)=0$, control space $U=(-\infty, \infty)$, and functional $I=g=y(1)$. We seek the minimum of $I$ in the class $\Omega$ of all admissible systems $x(t), y(t), u(t), 0 \leqslant t \leqslant 1$, satisfying

$$
|u(t)| \leqslant 2 \epsilon^{-1} t^{-1 / 2}+\epsilon t u^{2}(t)
$$

for almost all $t$ and any $0<\epsilon \leqslant 1$. The class $\Omega$ is nonempty since

$$
x(t)=1-t, \quad y(t)=t^{2} / 2, \quad u(t)=-1, \quad 0 \leqslant t \leqslant 1,
$$

is an admissible system and satisfies $\left(\gamma^{\prime}\right)$. Here, we have $H=t u^{2}$, $M_{\mathrm{\varepsilon}}=2 \epsilon^{-1} t^{-1 / 2}$. For the minimum of $I$, it suffices to consider the subclass $\Omega_{0}$ of all systems $x, y, u$ of $\Omega$, with $0 \leqslant I=y(1) \leqslant 1 / 2$; hence,

$$
\int_{0}^{1} H d t=\int_{0}^{1} t u^{2} d t=y(1) \leqslant 1 / 2=\eta .
$$


Then, $0 \leqslant y(t) \leqslant y(1) \leqslant 1 / 2=\eta$, and, if $\epsilon=1$, also

$$
|x(t)|<1+\int_{0}^{1}\left(2 \tau^{-1 / 2}+\tau u^{2}\right) d \tau<1+4+\eta=\delta,
$$

and it suffices to consider the compact set

$$
A_{0}=[0 \leqslant t \leqslant 1,|x| \leqslant \delta, 0 \leqslant y \leqslant \eta] .
$$

All conditions of the existence theorem above are satisfied, and the problem possesses an absolute minimum. Note that the relation $\left(\gamma^{\prime}\right)$ expresses condition (4) of the existence theorem. If in place of $\left(\gamma^{\prime}\right)$ we use the relation

$$
\int_{0}^{1}|u(t)|^{2} d t \leqslant M \quad \text { for some } \quad M \geqslant 1,
$$

then we can use condition (3) of the existence theorem. It is well known that the problem has no absolute minimum without any relation $\left(\gamma^{\prime}\right)$ or $\left(\gamma^{\prime \prime}\right)$.

Example 4.3. As a third example, we consider the control problem with differential equations $d x / d t=u, d y / d t=u^{2}, 0 \leqslant t \leqslant 1$, boundary conditions $x(0)=0, y(0)=0, x(1)=1$, control space $U=(-\infty<u<\infty)$, and functional $I=g=y(1)$. We seek the minimum of $I$ in the class $\Omega$ of all admissible systems $x(t), y(t), u(t), 0 \leqslant t \leqslant 1$, satisfying

$$
|u(t)| \leqslant 10^{-2} \epsilon^{-2}+\epsilon u^{2}(t)
$$

for almost all $t$ and any $0<\epsilon \leqslant 1$. This example is modeled on the one of Remark 4.1. The same argument as in Example 4.2 shows that this examplehas an absolute minimum. Note that there is a choice of the function $M_{i \epsilon}(t)$ such that a condition $\left(\gamma^{\prime}\right)$ is always satisfied without restricting the class of the admissible pairs. Indeed, $|u| \leqslant \epsilon^{-2}+\epsilon u^{2}$ for all $u \in(-\infty,+\infty)$ and $0<\epsilon \leqslant 1$. This can be seen by noting that, for $|u| \leqslant \epsilon^{-1}$, we have $|u| \leqslant \epsilon^{-1}+\epsilon u^{2}$; for $|u| \geqslant \epsilon^{-1}$, we have $|u| \leqslant \epsilon|u||u| \leqslant \epsilon^{-2}+\epsilon u^{2}$.

In Example 4.1, we could determine a function $M_{i \epsilon}(t)$ such that relation $\left(\gamma^{\prime}\right)$ is always satisfied. In Example 4.2, no such function exists, and actually the problem has no absolute minimum; but, by suitably restricting the class $\Omega$ by means of a condition $\left(\gamma^{\prime}\right)$, we obtained a problem to which the existence theorem applies, and the absolute minimum in the restricted class exists. In Example 4.3, a relation $\left(\gamma^{\prime}\right)$ is always satisfied by a suitable $M_{i \epsilon}(t)$, but we have restricted the class $\Omega$ by a condition $\left(\gamma^{\prime \prime \prime}\right)$. 


\section{Proof of Existence Theorem}

The proof is essentially the same as for Theorem A in Ref. 1 but for the remarks below. First, instead of the only auxiliary variable $x^{0}$ as in the proof of Theorem $\mathrm{A}$ in Ref. 1 , we introduce $\gamma$ auxiliary variables $x^{n+1}, \ldots, x^{n+y}$ satisfying the differential euqations and initial conditions

$$
d x^{n+s} / d t=H_{s}(t, y(t), u(t)), \quad x^{n+s}\left(t_{1}\right)=0, \quad s=1, \ldots, \gamma .
$$

We use the notation $y=\left(x^{1}, \ldots, x^{\alpha}\right), z=\left(x^{\alpha+1}, \ldots, x^{n}\right), w=\left(x^{n+1}, \ldots, x^{n+y}\right)$. Let $y_{k}(t), z_{k}(t), u_{k}(t), t_{1 k} \leqslant t \leqslant t_{2 k}, k=1,2, \ldots$, be a minimizing sequence of systems of $\Omega$ and corresponding $w$, thus satisfying (2)-(4). We may well assume that we have already applied the selection process discussed in detail in Ref. 1, briefly, Ascoli's selection process on the components $x^{1}, \ldots, x^{\alpha}$ (that is, on the vector $y$ ) and Helly's selection process on the components $x^{\alpha+1}, \ldots, x^{n}$, $x^{n+1}, \ldots, x^{n+\gamma}$ (that is, on the vectors $z$ and $\left.w\right)$. Let $y(t), z(t), w(t), t_{1} \leqslant t \leqslant t_{2}$, be the limit vector function, so that $x_{k}(t) \rightarrow x^{i}(t)$ as $k \rightarrow \infty$ in the uniform topology if $i=1, \ldots, \alpha, x^{i}(t)$ absolutely continuous in $\left[t_{1}, t_{2}\right]$; and $x_{k}^{i}(t) \rightarrow x^{i}(t)$ as $k \rightarrow \infty$ pointwise if $i=\alpha+1, \ldots, n, n+1, \ldots, n+\gamma$, as proved in Ref. 1 . For $i=\alpha+1, \ldots, n, n+1, \ldots, n+\gamma$, let $x^{i}(t)=X^{i}(t)+S^{i}(t), t_{1} \leqslant t \leqslant t_{2}$, be the decomposition of $x^{i}$ as defined in Ref. $1, X^{i}$ absolutely continuous in $\left[t_{1}, t_{2}\right], S^{i}$ singular, $i=\alpha+1, \ldots, n+\gamma$. Then,

$$
X(t)=\left(x^{1}, \ldots, x^{\alpha}, X^{\alpha+1}, \ldots, X^{n}\right)=(y(t), Z(t)), t_{1} \leqslant t \leqslant t_{2},
$$

is a trajectory, that is, generated by a measurable strategy $u(t), t_{1} \leqslant t \leqslant t_{2}$, and $W(t)=\left(X^{n+1}, \ldots, X^{n+v}\right), t_{1} \leqslant t \leqslant t_{2}$, is the corresponding auxiliary vector $w$. The pairs $x_{k}(t), u_{k}(t), t_{1} \leqslant t \leqslant t_{2}, k=1,2, \ldots$, satisfy relations (2)-(4). Let us prove that $X(t), u(t), t_{1} \leqslant t \leqslant t_{2}$, also satisfy the same relations (2)-(4). In other words, we are going to prove a closure property of the class $\Omega$.

The statement concerning relations ( 3 ) for $i=1, \ldots, \beta$ is already contained in Ref. 1 and actually is a well-known consequence of the semicontinuity of the integral $\int\left|d x^{i} / d t\right|^{p} d t, p>1$, with respect to uniform topology. The statement concerning relations (2) for $s=1, \ldots, \gamma$ is already contained in Ref. 1, p. 538 , for a single function $H$ and only one auxiliary variable $x^{0}$. Its extension to $\gamma$ functions $H$ and $\gamma$ auxiliary variables $x^{n+1}, \ldots, x^{n+\gamma}$ is immediate. Let us prove now the statement concerning relations (4) for $i=\beta+1, \ldots, \alpha$. As in the proof of the closure theorem in Ref. 1, p. 528, let $\phi, \phi_{k}$ denote the $(n+\gamma)$-vectors

$$
\begin{aligned}
\phi(t) & =\left(y^{\prime}(t), Z^{\prime}(t), W^{\prime}(t)\right), \quad t_{1} \leqslant t \leqslant t_{2}, \\
\phi_{k a}(t) & =\left(y_{k i}^{\prime}(t), z_{k a}^{\prime}(t), w_{k}^{\prime}(t)\right), \quad t_{1 k} \leqslant t \leqslant t_{2 k}, \quad k=1,2, \ldots .
\end{aligned}
$$


As we proved in Ref. 1 for almost all $t \in\left[t_{1}, t_{2}\right]$, all these derivatives exist and equal the corresponding values of $f_{i}$ or $H_{s}$. Also, for almost every $t \in\left(t_{1}, t_{2}\right)$ and number $\eta>0$, there is some $h>0$, which we can choose as small as we want, and integer $\bar{k}, h=h(t, \eta), \bar{k}=\bar{k}(t, \eta, h)$, such that

$$
[t, t+h] \subset\left[t_{1}, t_{2}\right] \cap\left[t_{1 k}, t_{2 k}\right], \quad k \geqslant \bar{k},
$$

and

$$
\left|m_{h}-\phi(t)\right| \leqslant \eta, \quad\left|m_{h k}-m_{h}\right| \leqslant \eta, \quad k \geqslant k
$$

where

$$
\begin{gathered}
m_{h}=h^{-1} \int_{0}^{h} \phi(t+s) d s=\left(m_{h}^{1}, \ldots, m_{h}^{n+\gamma}\right), \\
m_{h k}=h^{-1} \int_{0}^{h} \phi_{k}(t+s) d s=\left(m_{h k}^{1}, \ldots, m_{h k}^{n+\gamma}\right), \quad k \geqslant \bar{k} .
\end{gathered}
$$

Let $\epsilon \in[\epsilon]$ and take $t$ having the properties mentioned above such that $M_{i \epsilon}(t)$ is the derivative of its integral for every $i=\beta+1, \ldots, \alpha$. Thus, for $i=\beta+1, \ldots, \alpha$ and $s=s(i)$, we have

$$
\begin{aligned}
& \left|x^{\prime i}(t)-m_{h}^{i}\right| \leqslant \eta, \quad\left|m_{h}^{i}-m_{h k}^{i}\right| \leqslant \eta, \\
& \left|x^{\prime n+s}(t)-m_{h}^{n+s}\right| \leqslant \eta, \quad\left|m^{n+s}-m_{h+s}^{n+s}\right| \leqslant \eta .
\end{aligned}
$$

On the other hand, the relation

$$
\left|f_{i}\left(\tau, y_{k}(\tau), u_{k}(\tau)\right)\right| \leqslant M_{i \xi}(\tau)+\epsilon H_{s}\left(\tau, y_{k}(\tau), u_{k}(\tau)\right)
$$

holds for almost all $\tau \in\left[t_{1 k}, t_{2 k}\right]$. Hence, by integration over $[t, t+h]$, we have

$$
\left|m_{h k}^{i}\right| \leqslant h^{-1} \int_{0}^{\hbar} M_{i \epsilon}(t+s) d s+\epsilon m_{h i}^{n+s}, \quad k \geqslant \bar{k} .
$$

We assume that $h$ is so small that

$$
\left|h^{-1} \int_{0}^{h} M_{i \epsilon}(t+s) d s-M_{i \epsilon}(t)\right| \leqslant \eta .
$$

By using (6)-(8), we obtain

$$
\begin{aligned}
\left|x^{i}(t)\right| & \leqslant\left|m_{h}^{i}\right|+\eta \leqslant\left|m_{h}^{i}\right|+2 \eta \\
& \leqslant 2 \eta+\left(M_{i \epsilon}(t)+\eta\right)+\epsilon\left(x^{n+s}(t)+2 \eta\right),
\end{aligned}
$$


or

$$
f_{i}(t, y(t), u(t)) \mid \leqslant M_{i \epsilon}(t)+\epsilon H_{s}(t, y(t), u(t))+(3+2 \epsilon) \eta, \quad i=\beta+1, \ldots, \alpha .
$$

Since $\eta>0$ is arbitrary and $\epsilon$ is fixed, (4) is proved for any given $\epsilon \in[\epsilon]$ and any $t \in\left(t_{1}, t_{2}\right)$ having the required properties, that is, for almost every $t \in\left[t_{1}, t_{2}\right]$. Every other part of the proof of Theorem $\mathrm{A}$ in Ref. 1 remains the same. The existence theorem above is thereby proved.

Remark 5.1. Existence Theorem B in Ref. 1 for usual optimal solutions, as well as Existence Theorems $\mathrm{A}^{*}, \mathrm{~B}^{*}$ in Ref. 1 for weak solutions, can be extended as for Theorem $\mathrm{A}$ above.

\section{References}

1. Cesari, L., Existence Theorems for Optimal Controls of the Mayer Type, SIAM Journal on Control, Vol. 6, No. 4, 1968.

2. CESARI, L., Existence Theorem for Weak and Usual Optimal Solutions in Lagrange Problems with Unilaternal Constraints, $I$ and II, Transactions of the American Mathematical Society, Vol. 124, No. 3, 1966.

3. Nishiura, T., On an Existence Theorem for Optimal Control, SIAM Journal on Control, Vol. 5, No. 4, 1967.

4. McShane, E. J., Relaxed Controls and Variational Problems, SIAM Journal on Control, Vol. 5, No. 3, 1967. 